\title{
Associations between cytokine gene polymorphisms and rheumatoid arthritis in Turkish population
}

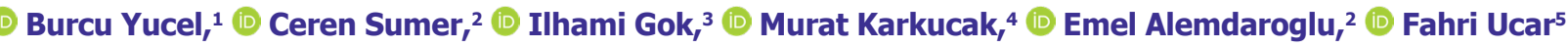 \\ ${ }^{1}$ Department of Medical Biology, Istanbul Medeniyet University Faculty of Medicine, Istanbul, Turkey \\ 2Department of Medical Biology, Karadeniz Technical University Faculty of Medicine, Trabzon, Turkey \\ ${ }^{3}$ Department of Bioengineering, Kafkas University Faculty of Engineering and Architecture, Kars, Turkey \\ 4Division of Rheumatology, Department of Physical Medicine and Rehabilitation, Karadeniz Technical University, Farabi Hospital, Trabzon, Turkey \\ ${ }^{5}$ Department of Medical Biology and Genetics, Akdeniz University Faculty of Medicine, Antalya, Turkey
}

\begin{abstract}
OBJECTIVE: Various cytokine polymorphisms have been associated with genetic risk factors predisposing to Rheumatoid Arthritis (RA) in different populations. To predict the clinical outcome as well as response to therapy in RA, studies aimed to describe genetic markers. The present study aims to search for polymorphisms of 13 cytokine coding genes in the Eastern Black Sea Region of Turkey.

METHODS: DNAs of 49 patients and 96 healthy bone marrow and kidney donors were isolated from peripheral blood samples. Genotyping was performed using the Heidelberg Cytokine Typing Tray kit. PCR products were visualized on an agarose gel, and results were analyzed using the interpretation scheme provided with the kit. Arlequin 3.5 software was used for statistical analysis.

RESULTS: No positive association was found between allele frequencies and the disease. However, a negative association was found for the IL-A $-889 \mathrm{C}$ allele $(\mathrm{p}=0.02, \mathrm{OR}=0.533$, Wald's $95 \% \mathrm{CI}=0.318-0.893)$. IL-12 $-1188 \mathrm{CC}(\mathrm{p}=0.01, \mathrm{OR}=3.667$, Wald's $95 \% \mathrm{CI}=1.246-10.786)$, IL-4 $-1098 \mathrm{GT}(\mathrm{p}=0.02, \mathrm{OR}=2.405$, Wald's $95 \% \mathrm{CI}=1.129-5.125)$ genotypes were found positively associated with the RA, while IL-4 $-590 \mathrm{CT}(\mathrm{p}=0.02$, OR $=0.422$, Wald's $95 \% \mathrm{CI}=0.201-0.886$ ) was found negatively associated with the disease. In addition, IL- 6 GG haplotype was found positively associated with the RA $(p=0.02, O R=1.880$, Wald's 95\% CI $=1.086-3.254$ ).
\end{abstract}

CONCLUSION: Our findings suggest that some polymorphisms of the IL-1A, IL-2, IL-4, IL-6 and IL-12 could be responsible for the susceptibility or protective to RA in our study population. Multi-centered and large numbers of subjects containing studies that search for cytokine polymorphisms will gather more information regarding the susceptibility to RA of Turkish patients.

Keywords: Cytokine polymorphism; PCR-SSP; rheumatoid arthritis.

Cite this article as: Yucel B, Sumer C, Gok I, Karkucak M, Alemdaroglu E, Ucar F. Associations between cytokine gene polymorphisms and rheumatoid arthritis in Turkish population. North Clin Istanb 2020;7(6):563-571.

$\mathrm{R}^{\mathrm{h}}$ heumatoid Arthritis (RA [MIM 180300]) is a chronic, painful, systemic, inflammatory, autoimmune disease with unknown etiology and it is characterized by the proliferation of synovial cells, dysfunction of joints [1]. The prevalence of the disease is almost $1 \%$ worldwide and the Female/Male ratio is 2-3/1 [2]. Pain, joint deformity, physical dysfunction decrease the life quality of patients and the rheumatoid factor is found positive in $75-80 \%$ of the patients [3]. According to the common view accepted today, the disease of RA begins with the antigenic stimulation of the environmental factors that we do not yet know exactly in genetically predisposed individuals and is

Received: December 16, 2019 Accepted: June 09, 2020 Online: November 11, 2020

Correspondence: Burcu YUCEL, MD. Istanbul Medeniyet Universitesi Tip Fakultesi, Tibbi Biyoloji Anabilim Dali, Istanbul, Turkey.

Tel: +9021628023 31 e-mail: burcu_yucel@msn.com

(c) Copyright 2020 by Istanbul Provincial Directorate of Health - Available online at www.northclinist.com 
considered to be chronic with complex interactions in the immune system [4]. In the etiology of RA, genetic, immune system disorder, infectious agents, trauma, gender, stress, endocrine, metabolic, environmental, psychologi$\mathrm{cal}$ and nutritional factors are thought to play a role. Although these factors affect the course of the disease, none of these factors can be shown as a definite cause alone. However, the imbalance between pro-inflammatory and anti-inflammatory cytokines released from macrophages, monocytes and synovial fibroblasts is speculated to play a role in the inflammation of joints [5]. In addition to Class II MHC genes belonging to human leukocyte antigen (HLA) loci, multiple cytokine polymorphisms have been implicated as genetic risk factors predisposing to RA [4]. Cytokine polymorphisms have been linked to many inflammatory, infectious and autoimmune diseases and it is now known that polymorphisms in regulatory regions of the cytokine genes affect their secretion [6]. Inheritance of the polymorphisms is influenced by the ethnicity and ethnic differences in patterns of cytokine gene polymorphisms have been associated with predisposition to various diseases in different populations [7]. Online databases have been designed to promote efforts in search of associations between the diversity of polymorphisms and diseases [8]. The main and common aim of these efforts is to describe immunogenic markers for diseases to predict the clinical outcome as well as response to therapy. Therefore, this study aims to evaluate allele, genotype and haplotype frequencies of single nucleotide polymorphisms (SNP) of 13 cytokine and receptor genes in patients with $\mathrm{RA}$ in the Eastern Black Sea region of Turkey.

\section{MATERIALS AND METHODS}

\section{Study Group}

This study was conducted using DNA samples of 49 patients (mean age $52 \pm 12$ ) who were diagnosed with Rheumatoid Arthritis according to the modified ARA criteria in the Department of Physical Medicine and Rehabilitation in Karadeniz Technical University (KTU) Medical Faculty, Farabi Hospital between February 2008-May 2009. Control group consists of 96 healthy bone marrow and kidney donors for the bone marrow and kidney transplant patients with no history of RA. This study was supported by KTU BAP (project \# 2007.114.001.6) and approved by the Ethics Committee of Faculty of Medicine (2008/55). This study was conducted in 2009. Statistical analysis, data evaluation and writing were completed in 2019.

\section{DNA Isolation and Genotyping}

DNA was isolated from peripheral blood samples of 49 RA patients and 96 healthy controls using Bio-robot EZ1 magnetic bead-based workstation (QIAGEN, Hilden, Germany). Polymerase chain reaction with sequence specific primers (PCR-SSP) was performed for genotyping using Heidelberg Cytokine Typing Tray kit (University of Heidelberg, Heidelberg, Germany) according to manufacturer's instructions. Briefly, $2.5 \mu \mathrm{g}$ DNA mixed with a master mix containing 20 U Gotaq flexi taq polymerase (Promega, USA), enzyme buffer, $\mathrm{MgCl}_{2}$ at a $0.5 \mathrm{mM}$ final concentration. Then, $25 \mu \mathrm{l}$ of the master mix was distributed to each well of a primer consisting 96 well plate kit tray. PCR condition was as follows; initial denaturation $94^{\circ} \mathrm{C}, 2 \mathrm{~min}$; denaturation $94^{\circ} \mathrm{C}, 10$ seconds; annealing + extension $65^{\circ} \mathrm{C}, 1 \mathrm{~min}$ ute $\left(10\right.$ cycles); denaturation $94^{\circ} \mathrm{C}, 10$ seconds; annealing $61^{\circ} \mathrm{C}$, 50 seconds; extension $72^{\circ} \mathrm{C}, 30$ seconds $(20$ cycles). PCR products were visualized on $2 \%$ agarose gel. Results were analyzed using the interpretation scheme provided with the kit.

\section{Statistical Analysis}

Allele frequencies and Hardy Weinberg proportions (HWP) for each SNP were determined using the Arlequin 3.5 software [9]. Comparisons of different genotypes for two groups were tested using the $\chi^{2}$ test with Yates correction and Fisher's exact test. Crude odds ratios (OR), as estimates of the relative risk, were calculated within 95\% CI and Bonferroni correction test was applied for multiple comparisons. A value of $p<0.025$ was considered statistically significant.

\section{RESULTS}

\section{Allele Frequencies}

Allele frequencies for each SNP of 49 RA patients and 96 healthy controls are presented in Table 1 . We did not find any positive association between allele frequencies and the disease. However, we found negative association for the IL-1A $-889 \mathrm{C}$ allele $(\mathrm{p}=0.02, \mathrm{OR}=0.533$, Wald's $95 \% \mathrm{CI}=0.318-0.893)$.

\section{Genotype Frequencies}

Genotype frequencies of the patients and the healthy controls are given in Table 2. Positive associations between IL-12 $-1188 \mathrm{CC}(\mathrm{p}=0.01, \mathrm{OR}=3.667$, Wald's 95\% CI=1.246-10.786), IL-4 -1098 GT $(\mathrm{p}=0.02$, 
TABLE 1. Cytokine allele frequency, Pearson's p-value, odds ratio, and Wald's $95 \%$ confidence interval in RA patients and healthy controls

\begin{tabular}{|c|c|c|c|c|c|c|c|c|}
\hline \multirow[t]{2}{*}{ Cytokine polymorphism } & \multirow[t]{2}{*}{ Allele } & \multicolumn{2}{|c|}{$\mathrm{RA}(\mathrm{n}=49)$} & \multicolumn{2}{|c|}{ Control $(n=96)$} & \multirow[t]{2}{*}{ Pearson's p-value } & \multirow[t]{2}{*}{ OR } & \multirow[t]{2}{*}{ Wald' $95 \% \mathrm{CI}$} \\
\hline & & $\mathrm{N}$ & $\mathrm{F}$ & $\mathrm{N}$ & $\mathrm{F}$ & & & \\
\hline \multirow[t]{2}{*}{ IL-1A } & $\mathrm{C}$ & 59 & 0.60 & 142 & 0.74 & $0.02^{\mathrm{b}}$ & 0.533 & $0.318-0.893$ \\
\hline & $\mathrm{T}$ & 39 & 0.40 & 50 & 0.26 & & 1.877 & $1.119-3.149$ \\
\hline \multirow[t]{2}{*}{ IL-1 $\beta-511$} & C & 50 & 0.50 & 118 & 0.61 & 0.08 & 0.644 & $0.396-1.048$ \\
\hline & $\mathrm{T}$ & 50 & 0.50 & 76 & 0.39 & & 1.553 & $0.954-2.526$ \\
\hline \multirow[t]{2}{*}{ IL-1 $\beta+3962$} & $\mathrm{C}$ & 62 & 0.65 & 135 & 0.73 & 0.13 & 0.662 & $0.389-1.125$ \\
\hline & $\mathrm{T}$ & 34 & 0.35 & 49 & 0.27 & & 1.511 & $0.889-2.569$ \\
\hline \multirow[t]{2}{*}{$\mathrm{IL}-1 \mathrm{R}$} & $\mathrm{C}$ & 72 & 0.72 & 130 & 0.69 & 0.61 & 1.147 & $0.672-1.959$ \\
\hline & $\mathrm{T}$ & 28 & 0.28 & 58 & 0.31 & & 0.872 & $0.510-1.488$ \\
\hline \multirow{2}{*}{ IL-1RA } & $\mathrm{C}$ & 14 & 0.15 & 32 & 0.17 & 0.62 & 0.843 & $0.426-1.668$ \\
\hline & $\mathrm{T}$ & 82 & 0.85 & 158 & 0.83 & & 1.186 & $0.600-2.347$ \\
\hline \multirow[t]{2}{*}{ IL-4Ra } & $A$ & 84 & 0.84 & 164 & 0.85 & 0.75 & 0.896 & $0.460-1.748$ \\
\hline & G & 16 & 0.16 & 28 & 0.15 & & 1.116 & $0.572-2.176$ \\
\hline \multirow[t]{2}{*}{$\mathrm{IL}-12$} & $A$ & 58 & 0.58 & 130 & 0.69 & 0.06 & 0.616 & $0.372-1.019$ \\
\hline & $\mathrm{C}$ & 42 & 0.42 & 58 & 0.31 & & 1.623 & $0.981-2.685$ \\
\hline \multirow[t]{2}{*}{ g-IFN } & $A$ & 54 & 0.54 & 90 & 0.47 & 0.25 & 1.330 & $0.819-2.160$ \\
\hline & $\mathrm{T}$ & 46 & 0.46 & 102 & 0.53 & & 0.752 & $0.463-1.221$ \\
\hline \multirow[t]{2}{*}{ TGF-B codon 10} & $\mathrm{C}$ & 51 & 0.54 & 96 & 0.54 & 0.96 & 1.013 & $0.614-1.673$ \\
\hline & $\mathrm{T}$ & 43 & 0.46 & 82 & 0.46 & & 0.987 & $0.598-1.630$ \\
\hline \multirow[t]{2}{*}{ TGF-B codon 25} & $\mathrm{C}$ & 8 & 0.09 & 18 & 0.10 & 0.69 & 0.837 & $0.350-2.004$ \\
\hline & G & 86 & 0.91 & 162 & 0.90 & & 1.194 & $0.499-2.859$ \\
\hline \multirow[t]{2}{*}{ TNF- $\alpha-308$} & $A$ & 5 & 0.05 & 19 & 0.10 & 0.16 & 0.490 & $0.177-1.353$ \\
\hline & G & 93 & 0.95 & 173 & 0.90 & & 2.043 & $0.739-5.647$ \\
\hline \multirow[t]{2}{*}{ TNF- $\alpha-238$} & $A$ & 5 & 0.05 & 8 & 0.04 & 0.73 & 1.223 & $0.389-3.843$ \\
\hline & $\mathrm{G}$ & 93 & 0.95 & 182 & 0.96 & & 0.818 & $0.260-2.569$ \\
\hline \multirow[t]{2}{*}{ IL-2 -330 } & $\mathrm{G}$ & 44 & 0.45 & 96 & 0.50 & 0.41 & 0.815 & $0.500-1.328$ \\
\hline & $\mathrm{T}$ & 54 & 0.55 & 96 & 0.50 & & 1.227 & $0.753-2.000$ \\
\hline \multirow[t]{2}{*}{$\mathrm{IL}-2+160$} & $\mathrm{G}$ & 76 & 0.78 & 140 & 0.73 & 0.39 & 1.283 & $0.725-2.272$ \\
\hline & $\mathrm{T}$ & 22 & 0.22 & 52 & 0.27 & & 0.779 & $0.440-1.380$ \\
\hline \multirow[t]{2}{*}{ IL-4 -1098 } & G & 21 & 0.23 & 24 & 0.14 & 0.07 & 1.799 & $0.939-3.449$ \\
\hline & $\mathrm{T}$ & 71 & 0.77 & 146 & 0.86 & & 0.556 & $0.290-1.065$ \\
\hline IL-4 -590 & $\mathrm{C}$ & 74 & 0.80 & 122 & 0.71 & 0.09 & 1.685 & $0.914-3.105$ \\
\hline & $\mathrm{T}$ & 18 & 0.20 & 50 & 0.29 & & 0.594 & $0.322-1.094$ \\
\hline IL-4 -33 & $\mathrm{C}$ & 83 & 0.90 & 156 & 0.91 & 0.90 & 0.946 & $0.401-2.233$ \\
\hline & $\mathrm{T}$ & 9 & 0.10 & 16 & 0.09 & & 1.057 & $0.448-2.496$ \\
\hline IL-6 -174 & $\mathrm{C}$ & 33 & 0.34 & 78 & 0.41 & 0.27 & 0.752 & $0.451-1.254$ \\
\hline & G & 63 & 0.66 & 112 & 0.59 & & 1.330 & $0.798-2.216$ \\
\hline IL-6 nt565 & $A$ & 26 & 0.27 & 44 & 0.23 & 0.44 & 1.249 & $0.712-2.192$ \\
\hline & $\mathrm{G}$ & 70 & 0.73 & 148 & 0.77 & & 0.800 & $0.456-1.404$ \\
\hline IL-10 -1082 & A & 54 & 0.60 & 126 & 0.68 & 0.17 & 0.690 & $0.409-1.166$ \\
\hline & G & 36 & 0.40 & 58 & 0.32 & & 1.448 & $0.858-2.446$ \\
\hline IL-10 -819 & $\mathrm{C}$ & 66 & 0.72 & 115 & 0.62 & 0.10 & 1.567 & $0.912-2.694$ \\
\hline & $T$ & 26 & 0.28 & 71 & 0.38 & & 0.638 & $0.371-1.097$ \\
\hline IL-10 -592 & $A$ & 31 & 0.34 & 78 & 0.42 & 0.19 & 0.704 & $0.418-1.185$ \\
\hline & C & 61 & 0.66 & 108 & 0.58 & & 1.421 & $0.844-2.393$ \\
\hline
\end{tabular}

N: Absolute number; F: Frequency; OR: Odds ratio; CI: Confidence interval; b: Statistically significant after Bonferroni correction. 
TABLE 2. Cytokine genotype frequency, Pearson's p-value, odds ratio, and Wald's $95 \%$ confidence interval in RA patients and healthy controls

\begin{tabular}{|c|c|c|c|c|c|c|c|c|}
\hline \multirow[t]{2}{*}{ Cytokine polymorphism } & \multirow[t]{2}{*}{ Genotype } & \multicolumn{2}{|c|}{ RA $(n=49)$} & \multicolumn{2}{|c|}{ Control $(n=96)$} & \multirow[t]{2}{*}{ Pearson's p-value } & \multirow[t]{2}{*}{ OR } & \multirow[t]{2}{*}{ Wald' $95 \% \mathrm{CI}$} \\
\hline & & $\mathrm{N}$ & $\mathrm{F}$ & $\mathrm{N}$ & $\mathrm{F}$ & & & \\
\hline \multirow[t]{3}{*}{ IL-1A } & CC & 20 & 0.41 & 54 & 0.56 & 0.08 & 0.536 & $0.267-1.078$ \\
\hline & CT & 19 & 0.39 & 34 & 0.35 & 0.69 & 1.155 & $0.567-2.351$ \\
\hline & $\pi$ & 10 & 0.20 & 8 & 0.08 & 0.04 & 2.821 & $1.034-7.692$ \\
\hline \multirow[t]{3}{*}{ IL-1 $\beta-511$} & CC & 13 & 0.26 & 33 & 0.34 & 0.32 & 0.681 & $0.319-1.455$ \\
\hline & CT & 24 & 0.48 & 52 & 0.54 & 0.52 & 0.799 & $0.403-1.582$ \\
\hline & $\pi$ & 13 & 0.26 & 12 & 0.12 & 0.04 & 2.489 & $1.038-5.967$ \\
\hline \multirow[t]{3}{*}{ IL-1 $1 \beta+3962$} & CC & 22 & 0.46 & 54 & 0.59 & 0.15 & 0.595 & $0.295-1.203$ \\
\hline & CT & 18 & 0.38 & 27 & 0.29 & 0.33 & 1.444 & $0.691-3.018$ \\
\hline & $\pi$ & 8 & 0.17 & 11 & 0.12 & 0.44 & 1.473 & $0.549-3.949$ \\
\hline \multirow[t]{3}{*}{ IL-1R } & CC & 26 & 0.52 & 46 & 0.49 & 0.73 & 1.130 & $0.569-2.246$ \\
\hline & CT & 20 & 0.40 & 38 & 0.40 & 0.96 & 0.982 & $0.488-1.978$ \\
\hline & $\pi$ & 4 & 0.08 & 10 & 0.11 & 0.61 & 0.730 & $0.217-2.459$ \\
\hline \multirow[t]{3}{*}{ IL-1RA } & CC & 2 & 0.04 & 5 & 0.05 & $a$ & $\mathrm{a}$ & $\mathrm{a}$ \\
\hline & CT & 10 & 0.21 & 22 & 0.23 & 0.75 & 0.873 & $0.375-2.031$ \\
\hline & $\pi$ & 36 & 0.75 & 68 & 0.72 & 0.66 & 1.191 & $0.540-2.627$ \\
\hline \multirow[t]{3}{*}{ IL-4Ra } & $A A$ & 38 & 0.76 & 71 & 0.74 & 0.79 & 1.115 & $0.504-2.464$ \\
\hline & $A G$ & 8 & 0.16 & 22 & 0.23 & 0.33 & 0.641 & $0.262-1.566$ \\
\hline & GG & 4 & 0.08 & 3 & 0.03 & $\mathrm{a}$ & $\mathrm{a}$ & $a$ \\
\hline \multirow[t]{3}{*}{ IL-12 } & AA & 18 & 0.36 & 42 & 0.45 & 0.31 & 0.696 & $0.344-1.411$ \\
\hline & $A C$ & 22 & 0.44 & 46 & 0.49 & 0.57 & 0.820 & $0.411-1.634$ \\
\hline & $\mathrm{CC}$ & 10 & 0.20 & 6 & 0.06 & $0.01 b$ & 3.667 & $1.246-10.786$ \\
\hline \multirow[t]{3}{*}{ g-IFN } & $A A$ & 14 & 0.28 & 23 & 0.24 & 0.59 & 1.234 & $0.569-2.679$ \\
\hline & AT & 26 & 0.52 & 44 & 0.46 & 0.48 & 1.280 & $0.645-2.539$ \\
\hline & $\pi$ & 10 & 0.20 & 29 & 0.30 & 0.19 & 0.578 & $0.255-1.309$ \\
\hline \multirow[t]{3}{*}{ TGF-B codon 10} & CC & 12 & 0.26 & 20 & 0.22 & 0.69 & 1.183 & $0.519-2.694$ \\
\hline & CT & 27 & 0.57 & 56 & 0.63 & 0.53 & 0.796 & $0.387-1.635$ \\
\hline & $\pi$ & 8 & 0.17 & 13 & 0.15 & 0.71 & 1.199 & $0.458-3.137$ \\
\hline \multirow[t]{3}{*}{ TGF-B codon 25} & $\mathrm{CC}$ & 1 & 0.02 & 4 & 0.04 & $\mathrm{a}$ & $a$ & $a$ \\
\hline & CG & 6 & 0.13 & 10 & 0.11 & 0.77 & 1.171 & $0.398-3.447$ \\
\hline & GG & 40 & 0.85 & 76 & 0.84 & 0.92 & 1.053 & $0.393-2.818$ \\
\hline \multirow[t]{3}{*}{ TNF- $\alpha-308$} & AA & 1 & 0.02 & 2 & 0.02 & $a$ & $a$ & a \\
\hline & $A G$ & 3 & 0.06 & 15 & 0.16 & 0.10 & 0.352 & $0.097-1.281$ \\
\hline & GG & 45 & 0.92 & 79 & 0.82 & 0.12 & 2.421 & $0.767-7.639$ \\
\hline \multirow[t]{3}{*}{ TNF- $\alpha-238$} & $\mathrm{AA}$ & 1 & 0.02 & 0 & 0.00 & $a$ & $a$ & $a$ \\
\hline & $A G$ & 3 & 0.06 & 8 & 0.08 & $a$ & $a$ & a \\
\hline & GG & 45 & 0.92 & 87 & 0.92 & 0.96 & 1.034 & $0.295-3.622$ \\
\hline \multirow[t]{3}{*}{ IL-2 -330 } & GG & 6 & 0.12 & 23 & 0.24 & 0.10 & 0.443 & $0.167-1.173$ \\
\hline & GT & 32 & 0.65 & 50 & 0.52 & 0.13 & 1.732 & $0.85-3.528$ \\
\hline & $\pi$ & 11 & 0.22 & 23 & 0.24 & 0.84 & 0.919 & $0.405-2.083$ \\
\hline \multirow[t]{3}{*}{$\mathrm{IL}-2+166$} & GG & 32 & 0.65 & 54 & 0.56 & 0.29 & 1.464 & $0.718-2.987$ \\
\hline & GT & 12 & 0.24 & 32 & 0.33 & 0.27 & 0.649 & $0.298-1.411$ \\
\hline & $\pi$ & 5 & 0.10 & 10 & 0.10 & 0.97 & 0.977 & $0.315-3.035$ \\
\hline IL-4 -1098 & GG & 0 & 0.00 & 1 & 0.01 & $\mathrm{a}$ & $a$ & a \\
\hline
\end{tabular}




\begin{tabular}{|c|c|c|c|c|c|c|c|c|}
\hline \multirow[t]{2}{*}{ Cytokine polymorphism } & \multirow[t]{2}{*}{ Genotype } & \multicolumn{2}{|c|}{ RA $(n=49)$} & \multicolumn{2}{|c|}{ Control $(n=96)$} & \multirow[t]{2}{*}{ Pearson's p-value } & \multirow[t]{2}{*}{ OR } & \multirow[t]{2}{*}{ Wald' $95 \%$ CI } \\
\hline & & $\mathrm{N}$ & $\mathrm{F}$ & $\mathrm{N}$ & $\mathrm{F}$ & & & \\
\hline \multirow{5}{*}{ IL-4 -590 } & GT & 21 & 0.46 & 22 & 0.26 & $0.02 b$ & 2.405 & $1.129-5.125$ \\
\hline & $\pi$ & 25 & 0.54 & 62 & 0.73 & 0.03 & 0.442 & $0.208-0.937$ \\
\hline & CC & 29 & 0.63 & 37 & 0.43 & 0.03 & 2.259 & $1.083-4.713$ \\
\hline & CT & 16 & 0.35 & 48 & 0.56 & $0.02 b$ & 0.422 & $0.201-0.886$ \\
\hline & $\pi$ & 1 & 0.02 & 1 & 0.01 & $\mathrm{a}$ & $\mathrm{a}$ & $\mathrm{a}$ \\
\hline \multirow[t]{3}{*}{ IL-4 -33 } & CC & 38 & 0.83 & 71 & 0.83 & 0.99 & 1.004 & $0.390-2.580$ \\
\hline & CT & 7 & 0.15 & 14 & 0.16 & 0.87 & 0.923 & $0.344-2.478$ \\
\hline & $\pi$ & 1 & 0.02 & 1 & 0.01 & $a$ & $a$ & a \\
\hline \multirow[t]{3}{*}{ IL-6 -174 } & $\mathrm{CC}$ & 4 & 0.08 & 12 & 0.13 & 0.44 & 0.629 & $0.191-2.065$ \\
\hline & CG & 25 & 0.52 & 54 & 0.57 & 0.59 & 0.825 & $0.411-1.657$ \\
\hline & GG & 19 & 0.40 & 29 & 0.31 & 0.28 & 1.491 & $0.722-3.078$ \\
\hline \multirow[t]{3}{*}{ IL-6 nt565 } & $A A$ & 1 & 0.02 & 5 & 0.05 & $a$ & $a$ & a \\
\hline & $A G$ & 24 & 0.50 & 34 & 0.35 & 0.09 & 1.824 & $0.902-3.685$ \\
\hline & GG & 23 & 0.48 & 57 & 0.59 & 0.19 & 0.629 & $0.313-1.265$ \\
\hline \multirow[t]{3}{*}{ IL-10 -1082 } & $\mathrm{AA}$ & 11 & 0.24 & 39 & 0.42 & 0.04 & 0.440 & $0.198-0.974$ \\
\hline & $A G$ & 32 & 0.71 & 48 & 0.52 & 0.03 & 2.256 & $1.052-4.842$ \\
\hline & GG & 2 & 0.04 & 5 & 0.05 & $a$ & $a$ & $a$ \\
\hline \multirow[t]{3}{*}{ IL-10 -819 } & $\mathrm{CC}$ & 24 & 0.52 & 37 & 0.40 & 0.17 & 1.651 & $0.810-3.365$ \\
\hline & CT & 18 & 0.39 & 41 & 0.44 & 0.58 & 0.815 & $0.397-1.675$ \\
\hline & $\pi$ & 4 & 0.09 & 15 & 0.16 & 0.23 & 0.495 & $0.154-1.588$ \\
\hline \multirow[t]{3}{*}{ IL-10 -592 } & AA & 4 & 0.09 & 10 & 0.11 & 0.70 & 0.790 & $0.234-2.671$ \\
\hline & $A C$ & 23 & 0.50 & 58 & 0.62 & 0.16 & 0.603 & $0.295-1.233$ \\
\hline & $\mathrm{CC}$ & 19 & 0.41 & 25 & 0.27 & 0.09 & 1.914 & $0.909-4.031$ \\
\hline
\end{tabular}

N: Absolute number; F: Frequency; OR: Odds ratio; CI: Confidence interval; a: Cannot be calculated because expected $<5, \chi^{2}$ test; b: Statistically significant after Bonferroni correction.

$\mathrm{OR}=2.405$, Wald's $95 \% \mathrm{CI}=1.129-5.125)$, genotypes and the disease were found. On the other side, a negative association was found for IL-4 -590 CT $(\mathrm{p}=0.02$, $\mathrm{OR}=0.422$, Wald's $95 \% \mathrm{CI}=0.201-0.886)$ genotype.

\section{Haplotype Frequencies}

TGF- $\beta 1$, TNF- $\alpha$, IL- 2 , IL-4, IL- 6, IL- 10 genes have multiple SNPs and we therefore detected the haplotype frequencies of these genes. Haplotype frequencies of the patients and the healthy people are presented in Table 3 . The findings showed that the IL-6 GG haplotype was positively associated with RA ( $p=0.02, O R=1.880$, Wald's $95 \% \mathrm{CI}=1.086-3.254)$. We did not find statistically significant negative associations between haplotypes and the disease in our study group.

\section{Cytokine Diplotypes}

Combinations of haplotypes from both parents (haplotype zygosity or cytokine diplotypes are presented in Table 4. Our results suggest that IL-2 TG:GG $(\mathrm{p}=0.04, \mathrm{OR}=0.267$, Wald's 95\% CI=0.071-1.013) cytokine diplotype is positively associated with RA. On the other side, no significant negative association was found.

\section{DISCUSSION}

Expression of various cytokines that regulate immune responses is claimed to be associated with the pathogenesis of the RA [6]. It has been shown that several cytokine gene polymorphisms regulate their production in joints and linked to disease onset and/or progression 


\begin{tabular}{|c|c|c|c|c|c|c|c|c|}
\hline \multirow[t]{2}{*}{ Cytokine polymorphism } & \multirow[t]{2}{*}{ Haplotype } & \multicolumn{2}{|c|}{ RA $(n=49)$} & \multicolumn{2}{|c|}{ Control $(n=96)$} & \multirow[t]{2}{*}{ Pearson's p-value } & \multirow[t]{2}{*}{ OR } & \multirow[t]{2}{*}{ Wald' $95 \%$ CI } \\
\hline & & N & $\mathrm{F}$ & $\mathrm{N}$ & $\mathrm{F}$ & & & \\
\hline \multirow[t]{3}{*}{ TGF- $\beta$} & CC & 6 & 0.07 & 6 & 0.04 & 0.44 & 1.576 & $0.492-5.048$ \\
\hline & CG & 44 & 0.48 & 62 & 0.44 & 0.54 & 1.178 & $0.694-2.000$ \\
\hline & TG & 41 & 0.45 & 72 & 0.51 & 0.34 & 0.774 & $0.456-1.315$ \\
\hline \multirow[t]{4}{*}{ TNF- $\alpha$} & $A A$ & 2 & 0.02 & 0 & 0.00 & $a$ & $\mathrm{a}$ & $a$ \\
\hline & AG & 3 & 0.03 & 15 & 0.08 & 0.10 & 0.364 & $0.103-1.290$ \\
\hline & GA & 2 & 0.02 & 5 & 0.03 & $a$ & $\mathrm{a}$ & $\mathrm{a}$ \\
\hline & GG & 91 & 0.93 & 168 & 0.89 & 0.34 & 1.548 & $0.631-3.798$ \\
\hline \multirow[t]{4}{*}{ IL-2 } & GG & 41 & 0.44 & 87 & 0.45 & $a$ & $\mathrm{a}$ & $a$ \\
\hline & GT & 1 & 0.01 & 9 & 0.05 & 0.12 & 0.221 & $0.028-1.771$ \\
\hline & TG & 32 & 0.34 & 55 & 0.29 & 0.32 & 1.307 & $0.769-2.220$ \\
\hline & $\pi$ & 19 & 0.20 & 41 & 0.21 & 0.86 & 0.946 & $0.513-1.742$ \\
\hline \multirow[t]{3}{*}{ IL-4 } & GCC & 11 & 0.14 & 16 & 0.15 & 0.91 & 0.952 & $0.415-2.186$ \\
\hline & TCC & 60 & 0.79 & 75 & 0.71 & 0.21 & 1.550 & $0.776-3.097$ \\
\hline & $\pi T$ & 5 & 0.07 & 15 & 0.14 & 0.11 & 0.427 & $0.148-1.231$ \\
\hline \multirow[t]{3}{*}{ IL-6 } & $\mathrm{CA}$ & 18 & 0.21 & 45 & 0.28 & 0.27 & 0.703 & $0.376-1.313$ \\
\hline & CG & 10 & 0.12 & 33 & 0.20 & 0.09 & 0.524 & $0.244-1.124$ \\
\hline & GG & 56 & 0.67 & 83 & 0.52 & $0.02 b$ & 1.880 & $1.086-3.254$ \\
\hline \multirow[t]{3}{*}{ IL-10 } & ATA & 33 & 0.36 & 64 & 0.41 & 0.40 & 0.795 & $0.467-1.355$ \\
\hline & ACC & 22 & 0.24 & 41 & 0.26 & 0.66 & 0.874 & $0.481-1.588$ \\
\hline & GCC & 37 & 0.40 & 50 & 0.32 & 0.21 & 1.413 & $0.827-2.414$ \\
\hline
\end{tabular}

$\mathrm{N}$ : Absolute number; F: Frequency; OR: Odds ratio; CI: Confidence interval; a: Cannot be calculated because expected $<5, \chi^{2}$ test; b: Statistically significant after Bonferroni correction.

of the RA [3]. Additionally, genetic polymorphisms of these cytokines have been proposed to have an association with the susceptibility to the disease [10].

IL-1 cytokine expression increases the osteoclast activation and thus considered as a major contributor to RA [11]. IL-1a and IL-1 $\beta$ are proinflammatory cytokines and IL-1 receptor antagonist (IL-1RA) is an anti-inflammatory protein. There is a balance between these three cytokines and IL-1RA inhibits the receptor binding of IL-1A and IL-1 $\beta$ [12]. In various populations, single nucleotide polymorphisms (SNPs) of the genes encoding these three proteins have been associated with the susceptibility/protection to the RA [13]. IL-1A gene polymorphisms $-511 \mathrm{C} / \mathrm{T}$ and in exon 5 at +4845 $\mathrm{G} / \mathrm{T}$ ) also influence the IL-1 expression and was found to be associated susceptibility to RA [4]. In this study, we found that IL-1A-889 C allele could be associated with the susceptibility of RA $(p \leq 0.025)$ in the population of Eastern Black Sea Region of Turkey. A meta-analysis study concluded 16 studies with 4339 RA patients showed no significant association between RA susceptibility and IL-1A -889 C/T allele [14].

Interleukin-4 (IL-4) promotes the generation of Th2 cells by differentiation of naive $T$ cells, then activated Th2 cells produce extra IL-4 by positive-feedback [3]. IL-4 gene polymorphism -590 T:C has been found to increase the risk of RA in Europeans [15] and Chinese [16]. Thus, it was suggested as a genetic marker for the evaluation of the susceptibility and severity of RA [17]. A susceptible association was found for IL-4 -590/C:C and -1098 TT homozygous genotypes in population of ethnic Macedonians [18]. On the contrary, in our study group, we found a positive association for- 1098 GT genotype. Furthermore, frequencies of IL-4 -590 CT were found higher in healthy controls $(p \leq 0.025)$ suggesting a presumptive protective effect for RA in our study population.

IL-12 has been shown to play a role in bone erosion by activating nuclear factor kappa light chain enhancer of activated $B$ cells $(N F-\kappa B)$ in a RANKL dependent 
TABLE 4. Cytokine diplotypes (haplotype zygotes), Pearson's p-value, odds ratio, and Wald's 95\% confidence interval in RA patients and healthy controls

\begin{tabular}{|c|c|c|c|c|c|c|c|c|}
\hline \multirow[t]{2}{*}{ Cytokine polymorphism } & \multirow[t]{2}{*}{ Genotype } & \multicolumn{2}{|c|}{ RA $(n=49)$} & \multicolumn{2}{|c|}{ Control $(n=96)$} & \multirow[t]{2}{*}{ Pearson's p-value } & \multirow[t]{2}{*}{ OR } & \multirow[t]{2}{*}{ Wald' $95 \% \mathrm{CI}$} \\
\hline & & $\mathrm{N}$ & $\mathrm{F}$ & $\mathrm{N}$ & $\mathrm{F}$ & & & \\
\hline \multirow[t]{6}{*}{ TGF- $\beta$} & $\mathrm{CC}: \mathrm{CC}$ & 1 & 0.02 & 2 & 0.03 & a & a & a \\
\hline & CC:TG & 4 & 0.09 & 2 & 0.03 & $a$ & $a$ & a \\
\hline & CG:CG & 10 & 0.21 & 7 & 0.10 & 0.08 & 2.510 & $0.881-7.149$ \\
\hline & CG:TC & 3 & 0.06 & 4 & 0.06 & $\mathrm{a}$ & $\mathrm{a}$ & $a$ \\
\hline & CG:TG & 21 & 0.45 & 44 & 0.61 & 0.08 & 0.514 & $0.244-1.083$ \\
\hline & TG:TG & 8 & 0.17 & 13 & 0.18 & 0.88 & 0.931 & $0.353-2.454$ \\
\hline \multirow[t]{5}{*}{ TNF- $\alpha$} & $A A: A A$ & 1 & 0.02 & 0 & 0.00 & $\mathrm{a}$ & $\mathrm{a}$ & a \\
\hline & GG:AG & 3 & 0.05 & 11 & 0.10 & 0.29 & 0.492 & $0.131-1.854$ \\
\hline & GG:GA & 2 & 0.04 & 5 & 0.04 & $\mathrm{a}$ & $\mathrm{a}$ & a \\
\hline & $A G: A G$ & 0 & 0.00 & 2 & 0.02 & $a$ & $a$ & a \\
\hline & GG:GG & 43 & 0.78 & 76 & 0.66 & 0.29 & 1.697 & $0.626-4.599$ \\
\hline \multirow[t]{10}{*}{ IL-2 } & GG:GG & 6 & 0.13 & 21 & 0.22 & 0.17 & 0.510 & $0.188-1.382$ \\
\hline & GG:GT & 1 & 0.02 & 1 & 0.01 & $a$ & $\mathrm{a}$ & $a$ \\
\hline & GG:TG & 1 & 0.02 & 0 & 0.00 & $a$ & $a$ & a \\
\hline & GG:TT & 4 & 0.08 & 17 & 0.18 & 0.13 & 0.422 & $0.158-1.129$ \\
\hline & TG:GG & 25 & 0.52 & 26 & 0.27 & $0.003^{b}$ & 2.926 & $1.42-6.033$ \\
\hline & TG:TG & 1 & 0.02 & 7 & 0.07 & $\mathrm{a}$ & $a$ & a \\
\hline & $\mathrm{TG}: \mathrm{TT}$ & 5 & 0.10 & 7 & 0.07 & a & a & $a$ \\
\hline & TG:GT & 0 & 0.00 & 8 & 0.08 & a & $a$ & a \\
\hline & TT:GG & 0 & 0.00 & 1 & 0.01 & a & a & $a$ \\
\hline & Tा:Tा & 5 & 0.10 & 8 & 0.08 & $a$ & $a$ & $\mathrm{a}$ \\
\hline \multirow[t]{17}{*}{ IL-4 } & TCC:GCC & 10 & 0.22 & 12 & 0.21 & 0.90 & 1.065 & $0.414-2.742$ \\
\hline & TCC:GCT & 1 & 0.02 & 0 & 0.00 & $a$ & $\mathrm{a}$ & a \\
\hline & TCC:GTC & 6 & 0.13 & 1 & 0.02 & a & a & $\mathrm{a}$ \\
\hline & TCC:GTT & 1 & 0.02 & 0 & 0.00 & $\mathrm{a}$ & $a$ & $a$ \\
\hline & TCC:TCC & 18 & 0.39 & 24 & 0.41 & 0.82 & 0.625 & $0.25-1.565$ \\
\hline & TTC:TTC & 0 & 0.00 & 2 & 0.03 & $a$ & $\mathrm{a}$ & $\mathrm{a}$ \\
\hline & TCC:TTC & 1 & 0.02 & 2 & 0.03 & a & a & $a$ \\
\hline & TTC:GCC & 1 & 0.02 & 0 & 0.00 & a & a & a \\
\hline & TTC:GCT & 1 & 0.02 & 0 & 0.00 & a & a & a \\
\hline & TTC:TCC & 2 & 0.04 & 0 & 0.00 & a & a & $a$ \\
\hline & TाT:GCT & 1 & 0.02 & 0 & 0.00 & $a$ & $a$ & a \\
\hline & TाT:TCC & 3 & 0.07 & 12 & 0.21 & 0.04 & 0.267 & $0.071-1.013$ \\
\hline & TाT:GCC & 0 & 0.00 & 2 & 0.03 & $a$ & $a$ & a \\
\hline & TCT:TTC & 0 & 0.00 & 1 & 0.02 & a & $a$ & $a$ \\
\hline & Tाт:ТCT & 0 & 0.00 & 1 & 0.02 & a & $a$ & $\mathrm{a}$ \\
\hline & GCC:GCC & 0 & 0.00 & 1 & 0.02 & a & a & $a$ \\
\hline & TाT:TTC & 1 & 0.02 & 0 & 0.00 & $a$ & a & $\mathrm{a}$ \\
\hline \multirow[t]{5}{*}{ IL-6 } & CA:CA & 1 & 0.02 & 6 & 0.06 & a & a & a \\
\hline & CG:CA & 2 & 0.04 & 7 & 0.07 & a & a & a \\
\hline & CG:CG & 1 & 0.02 & 0 & 0.00 & a & a & $\mathrm{a}$ \\
\hline & GC:GA & 2 & 0.04 & 0 & 0.00 & $a$ & $a$ & $\mathrm{a}$ \\
\hline & $\mathrm{GG}: \mathrm{CA}$ & 13 & 0.28 & 26 & 0.27 & 0.88 & 1.061 & $0.484-2.323$ \\
\hline
\end{tabular}


TABLE 4 (COnT.). Cytokine diplotypes (haplotype zygotes), Pearson's p-value, odds ratio, and Wald's 95\% confidence interval in RA patients and healthy controls

\begin{tabular}{|c|c|c|c|c|c|c|c|c|}
\hline \multirow[t]{2}{*}{ Cytokine polymorphism } & \multirow[t]{2}{*}{ Genotype } & \multicolumn{2}{|c|}{$\mathrm{RA}(\mathrm{n}=49)$} & \multicolumn{2}{|c|}{ Control $(n=96)$} & \multirow[t]{2}{*}{ Pearson's p-value } & \multirow[t]{2}{*}{ OR } & \multirow[t]{2}{*}{ Wald' $95 \% \mathrm{Cl}$} \\
\hline & & $\mathrm{N}$ & $\mathrm{F}$ & $\mathrm{N}$ & $\mathrm{F}$ & & & \\
\hline \multirow{20}{*}{ IL-10 } & GG:CG & 6 & 0.13 & 26 & 0.27 & 0.06 & 0.404 & $0.153-1.064$ \\
\hline & GG:GA & 4 & 0.09 & 0 & 0.00 & $a$ & $a$ & $\mathrm{a}$ \\
\hline & GG:GG & 16 & 0.35 & 31 & 0.32 & 0.77 & 1.118 & $0.532-2.35$ \\
\hline & GG:CA & 1 & 0.02 & 0 & 0.00 & $a$ & $a$ & a \\
\hline & ACA:ATA & 2 & 0.04 & 2 & 0.02 & $a$ & $a$ & $a$ \\
\hline & ACC:ACC & 1 & 0.02 & 2 & 0.02 & $a$ & $a$ & a \\
\hline & ACC:ACA & 0 & 0.00 & 2 & 0.02 & $a$ & a & a \\
\hline & ACC:ATA & 4 & 0.09 & 18 & 0.20 & a & a & a \\
\hline & ATA:ATA & 2 & 0.04 & 10 & 0.11 & a & a & a \\
\hline & ATC:ATA & 1 & 0.02 & 4 & 0.04 & a & a & a \\
\hline & GCC:ACA & 4 & 0.09 & 9 & 0.10 & $\mathrm{a}$ & $a$ & $\mathrm{a}$ \\
\hline & GCC:ACC & 16 & 0.36 & 17 & 0.18 & 0.03 & 2.434 & $1.087-5.45$ \\
\hline & GCC:ATA & 12 & 0.27 & 18 & 0.20 & 0.35 & 1.495 & $0.647-3.455$ \\
\hline & GCC:CCC & 1 & 0.02 & 0 & 0.00 & $a$ & $a$ & $\mathrm{a}$ \\
\hline & GCC:GCC & 2 & 0.04 & 4 & 0.04 & $a$ & $a$ & $a$ \\
\hline & GTC:ATA & 0 & 0.00 & 1 & 0.01 & a & $a$ & a \\
\hline & GCC:GCA & 0 & 0.00 & 1 & 0.01 & $a$ & $a$ & $a$ \\
\hline & GCC:ATC & 0 & 0.00 & 2 & 0.02 & a & a & a \\
\hline & GAA:ATA & 0 & 0.00 & 1 & 0.01 & a & a & a \\
\hline & CCC:AGA & 0 & 0.00 & 1 & 0.01 & $a$ & a & $\mathrm{a}$ \\
\hline
\end{tabular}

$\mathrm{N}$ : Absolute number; F: Frequency; OR: Odds ratio; CI: Confidence interval; a: Cannot be calculated because expected $<5, \chi^{2}$ test; b: Statistically significant after Bonferroni correction.

or independent manner [11]. Elevated levels of IL-12 were found in sera patients of RA patients [19]. No genetic associations have been found between Spanish RA patients and polymorphisms of IL-12B and IL-12RB1 [20]. However, in our study population, we found that IL-12 -1188 CC genotype was positively associated with RA significantly.

IL-6 gene polymorphisms have also been found associated with RA and the disease severity in some populations [21]. In this study, IL-6 GG haplotype frequency was found positively associated with RA $(\mathrm{p} \leq 0.025)$.

The size of the population included in this study is our limitation. We believe, multi-centered and large cohort studies searching for cytokine polymorphisms will gather more information regarding to susceptibility to RA of Turkish patients.

In conclusion, our findings suggest that some SNPs of the IL-1A, IL-2, IL-4, IL-6 and IL-12 could be responsi- ble for the susceptibility to RA in our study population. We found IL-4 -1098 TT, -590 GT, IL-10 -1082 AA genotypes might be protective for the RA. In addition to identifying the SNPs in cytokines, we believe that definition of cytokine networks and how SNPs deregulate these pathways in RA is more crucial in the induction of remission by blocking inflammation in the early stages of the disease.

Ethics Committee Approval: The Karadeniz Technical University Clinical Research Ethics Committee granted approval for this study (date: 08.01.2009, number: 2008/55).

Conflict of Interest: No conflict of interest was declared by the authors.

Financial Disclosure: Authors would like to thank KTU Scientific Research Fund.

Authorship Contributions: Concept - BY, FU; Design - BY; Supervision - FU; Fundings - FU; Materials - MK; Data collection and/or processing - BY, CS, IG, MK, EA; Analysis and/or interpretation - BY, CS; Literature review - BY; Writing - BY; Critical review - FU. 


\section{REFERENCES}

1. Kurkó J, Besenyei T, Laki J, Glant TT, Mikecz K, Szekanecz Z. Genetics of rheumatoid arthritis - a comprehensive review. Clin Rev Allergy Immunol 2013;45:170-9. [CrossRef]

2. Silman AJ, Pearson JE. Epidemiology and genetics of rheumatoid arthritis. Arthritis Res 2002;4 Suppl 3:S265-72. [CrossRef]

3. Rego-Pérez I, Fernández-Moreno M, Blanco FJ. Gene polymorphisms and pharmacogenetics in rheumatoid arthritis. Curr Genomics 2008;9:381-93. [CrossRef]

4. Bax M, van Heemst J, Huizinga TW, Toes RE. Genetics of rheumatoid arthritis: what have we learned? Immunogenetics 2011;63:459-66.

5. Okada Y, Wu D, Trynka G, Raj T, Terao C, Ikari K, et al. Genetics of rheumatoid arthritis contributes to biology and drug discovery. Nature 2014;506:376-81. [CrossRef]

6. McInnes IB, Schett G. Cytokines in the pathogenesis of rheumatoid arthritis. Nat Rev Immunol 2007;7:429-42. [CrossRef]

7. Ollier W, Thomson W. Population genetics of rheumatoid arthritis. Rheum Dis Clin North Am 1992;18:741-59.

8. Haukim N, Bidwell JL, Smith AJ, Keen LJ, Gallagher G, Kimberly R, et al. Cytokine gene polymorphism in human disease: on-line databases, supplement 2. Genes Immun 2002;3:313-30. [CrossRef]

9. Excoffier L, Lischer HE. Arlequin suite ver 3.5: a new series of programs to perform population genetics analyses under Linux and Windows. Mol Ecol Resour 2010;10:564-7. [CrossRef]

10. Mateen S, Zafar A, Moin S, Khan AQ, Zubair S. Understanding the role of cytokines in the pathogenesis of rheumatoid arthritis. Clin Chim Acta 2016;455:161-71. [CrossRef]

11. Magyari L, Varszegi D, Kovesdi E, Sarlos P, Farago B, Javorhazy A, et al. Interleukins and interleukin receptors in rheumatoid arthritis: Research, diagnostics and clinical implications. World J Orthop 2014;5:516-36. [CrossRef]

12. Isomäki P, Punnonen J. Pro- and anti-inflammatory cytokines in rheumatoid arthritis. Ann Med 1997;29:499-507. [CrossRef]

13. Lagha A, Zidi S, Stayoussef M, Gazouani E, Kochkar R, Kochbati S, et al. Interleukin-1 $\beta$, Interleukin1-Ra, Interleukin-10, and tumor necrosis factor- $\alpha$ polymorphisms in Tunisian patients with rheumatoid arthritis. Pathol Biol (Paris) 2015;63:179-84. [CrossRef]

14. Lee YH, Bae SC. Associations between interleukin-1 and IL-1 receptor antagonist polymorphisms and susceptibility to rheumatoid arthritis: A meta-analysis. Cell Mol Biol (Noisy-le-grand) 2015;61:105-11.

15. Barrera P, Fauré S, Prud'homme JF, Balsa A, Migliorini P, Chimenti D, et al; European Consortium on Rheumatoid Arthritis Families (ECRAF). European genetic study on rheumatoid arthritis: is there a linkage of the interleukin-1 (IL-1), IL-10 or IL-4 genes to RA? Clin Exp Rheumatol 2001;19:709-14.

16. Li X, Chai W, Ni M, Xu M, Lian Z, Shi L, et al. The effects of gene polymorphisms in interleukin-4 and interleukin- 6 on the susceptibility of rheumatoid arthritis in a Chinese population. Biomed Res Int 2014;2014:265435. [CrossRef]

17. Park HK, Kim SK, Kweon HY, Lee KG, Arasu MV, Kim YO. Promoter polymorphism $(-590, \mathrm{~T} / \mathrm{C})$ of interleukin 4 (IL4) gene is associated with rheumatoid arthritis: An updated meta-analysis. Saudi J Biol Sci 2017;24:444-9. [CrossRef]

18. Trajkov D, Mishevska-Perchinkova S, Karadzova-Stojanoska A, Petlichkovski A, Strezova A, Spiroski M. Association of 22 cytokine gene polymorphisms with rheumatoid arthritis in population of ethnic Macedonians. Clin Rheumatol 2009;28:1291-300. [CrossRef]

19. Kim W, Min S, Cho M, Youn J, Min J, Lee S, et al. The role of IL-12 in inflammatory activity of patients with rheumatoid arthritis (RA). Clin Exp Immunol 2000;119:175-81. [CrossRef]

20. Orozco G, González-Gay MA, Paco L, López-Nevot MA, Guzmán M, Pascual-Salcedo D, et al. Interleukin 12 (IL12B) and interleukin 12 receptor (IL12RB1) gene polymorphisms in rheumatoid arthritis. Hum Immunol 2005;66:710-5. [CrossRef]

21. Srirangan S, Choy EH. The role of interleukin 6 in the pathophysiology of rheumatoid arthritis. Ther Adv Musculoskelet Dis 2010;2:247-56. 